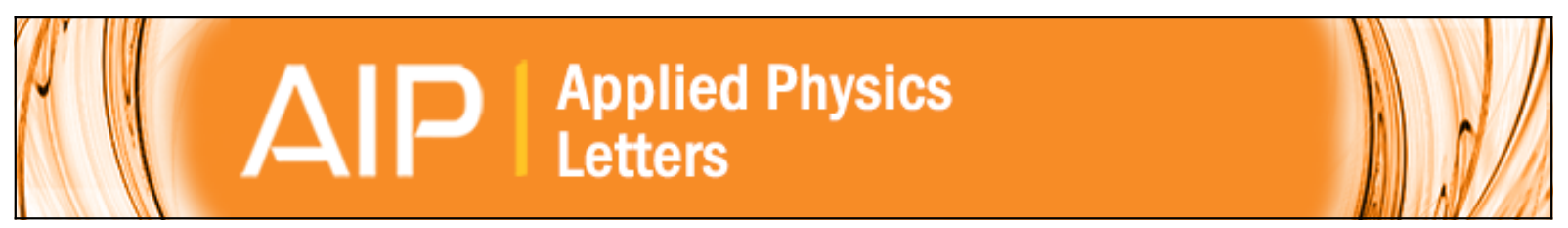

\title{
Acoustic Fresnel lenses with extraordinary transmission
}

Miguel Molerón, Marc Serra-Garcia, and Chiara Daraio

Citation: Applied Physics Letters 105, 114109 (2014); doi: 10.1063/1.4896276

View online: http://dx.doi.org/10.1063/1.4896276

View Table of Contents: http://scitation.aip.org/content/aip/journal/apl/105/11?ver=pdfcov

Published by the AIP Publishing

\section{Articles you may be interested in}

Extraordinary acoustic transmission mediated by Helmholtz resonators

AIP Advances 4, 077132 (2014); 10.1063/1.4891849

Experimental study on acoustic subwavelength imaging of holey-structured metamaterials by resonant tunneling J. Acoust. Soc. Am. 135, 1686 (2014); 10.1121/1.4868395

Extraordinary acoustic transmission through ultrathin acoustic metamaterials by coiling up space Appl. Phys. Lett. 103, 063509 (2013); 10.1063/1.4817925

Introduction to the Special Issue on Acoustic Metamaterials

J. Acoust. Soc. Am. 132, 2783 (2012); 10.1121/1.4751033

Super-resolution imaging by resonant tunneling in anisotropic acoustic metamaterials

J. Acoust. Soc. Am. 132, 2800 (2012); 10.1121/1.4744932

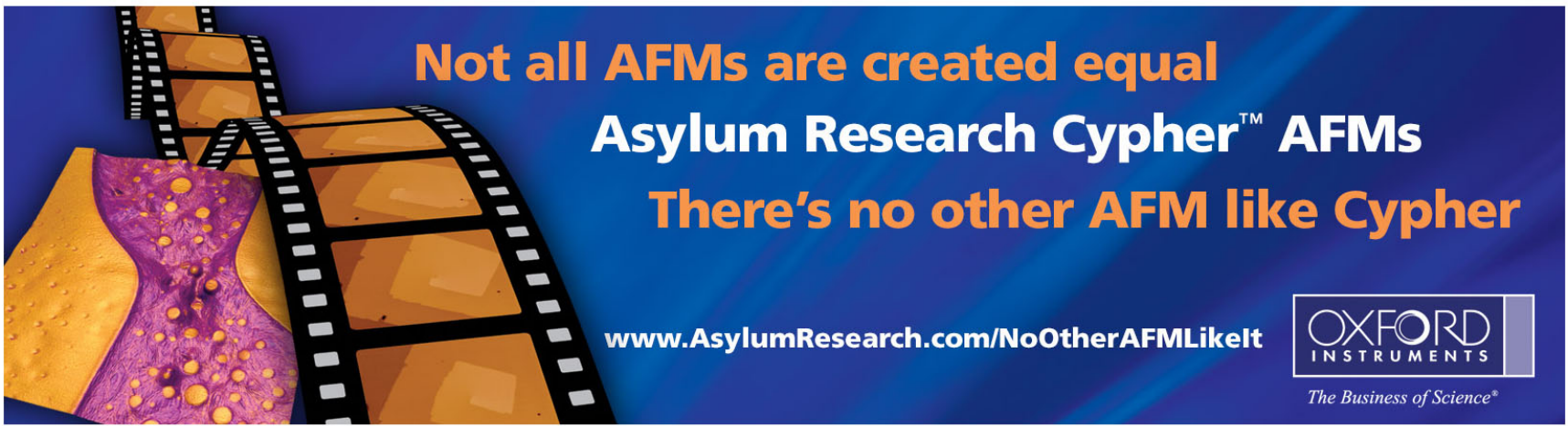




\title{
Acoustic Fresnel lenses with extraordinary transmission
}

\author{
Miguel Molerón, ${ }^{1, a)}$ Marc Serra-Garcia, ${ }^{1}$ and Chiara Daraio ${ }^{1,2}$ \\ ${ }^{1}$ Department of Mechanical and Process Engineering, ETH Zurich, CH-8092 Zurich, Switzerland \\ ${ }^{2}$ Division of Engineering and Applied Science, California Institute of Technology, Pasadena, \\ California 91125, USA
}

(Received 4 August 2014; accepted 10 September 2014; published online 19 September 2014)

\begin{abstract}
We investigate numerically and experimentally highly efficient acoustic lenses based on the principle of extraordinary acoustic transmission. We study circular, flat lenses composed of perforated air channels. The geometry is similar to binary Fresnel lenses, and the lenses exploit several resonance mechanisms to enhance the transmission, such as Fabry-Perot resonances in the channels and cavity resonances on the lens surface. The proposed lenses are able to transmit up to $83 \%$ of the incident energy and generate sharp focusing with very high amplification (up to $16 \mathrm{~dB}$ experimentally). Furthermore, the resulting lenses are thinner than other designs providing similar performance, making them ideal candidates for application in acoustic imaging and medical diagnostics. (C) 2014 AIP Publishing LLC. [http://dx.doi.org/10.1063/1.4896276]
\end{abstract}

Transmission efficiency is an important aspect in the design of acoustic lenses, particularly in the implementation of realistic devices. However, building highly efficient lenses proves challenging due to the lack of materials with high refractive index and low impedance mismatch with the host medium. To overcome this limitation, several designs have been proposed in the last decade based on phononic crystals (PCs) or acoustic metameterials. At wavelengths much smaller than the lattice constant, PCs can be considered as homogeneous materials with wave speed smaller than that of the host medium. Using this property, graded index (GRIN) lenses have been designed by varying the filling fraction of the PC in the transverse direction. ${ }^{1}$ At higher frequencies, acoustic focusing has also been achieved by exploiting the anisotropy of the equifrequency contours of PC. ${ }^{2}$ Using a different approach, aperiodic arrays of scatterers have been proposed to design efficient acoustic lenses. Håkanson et al. ${ }^{3,4}$ used genetic algorithms to optimise the amplification generated by two-dimensional (2D) aperiodic arrays of circular scatterers. Sanchis et al. ${ }^{5}$ extended the same idea to 3D axisymmetric lenses in which the optimized array is extruded around the central axis to form concentric rings. The interest of such a geometry is that energy is focused in a point (instead of a line in the 2D case), which leads to comparatively higher amplifications. The same idea has been applied to design PC axisymmetric GRIN lenses. ${ }^{6}$ Acoustic metamaterial GRIN lenses have also been proposed using crossshaped scatterers ${ }^{7}$ or coiled up space, ${ }^{8}$ providing high efficiency and smaller lens thickness compared to PC lenses.

Metamaterials are artificial materials in which the presence of resonances in the micro-/meso-structure leads to unprecedented properties, ${ }^{9}$ such as negative refractive index. ${ }^{10}$ Metamaterials consisting of a rigid plate (or opaque plate in the case of optical waves) with subwavelength apertures have been proposed for improving optical or acoustic transmission. A pioneering work by Ebbesen et al. ${ }^{11}$ demonstrated that such structure can transmit much more light than expected from

\footnotetext{
${ }^{\text {a) }}$ Author to whom correspondence should be addressed. Electronic mail: moleronm@ethz.ch
}

the classical aperture theory. ${ }^{12}$ This phenomenon, called extraordinary optical transmission (EOT), motivated a number of subsequent works (see Ref. 13 for a review) and was later extended to acoustic waves as extraordinary acoustic transmission (EAT). ${ }^{14,15}$ Theoretical studies ${ }^{16,17}$ have revealed that EAT is attributed to the interplay between Fabry-Perot resonances inside the holes and resonances of the surfaces waves propagating on the plate. More recently, a nonresonant and broadband mechanism to achieve EAT has been demonstrated by the existence of an acoustic Brewster angle. ${ }^{18}$

In our work, we investigate numerically and test experimentally the design of acoustic lenses based on the resonant EAT. We study circular, flat lenses with perforated air channels, in which different resonant mechanisms are used to enhance de transmission. The proposed lenses are able to transmit up to $83 \%$ of the incident energy and generate a sharp focal spot with very high amplitude. As discussed later, our lenses achieve $16 \mathrm{~dB}$ amplification in experiments. This amplification improves, by $8 \mathrm{~dB}$, the performance of GRIN lenses, ${ }^{6}$ and is slightly better than the amplification obtained with optimized aperiodic arrays. ${ }^{5}$ We study two different lens designs, as shown in Figs. 1(a) and 1(b), which will be referred to as Lens A and Lens B, respectively. Figs. 1(c) and 1(d) show the corresponding $3 \mathrm{D}$ printed lenses used in experiments. Lens A consists in a circular rigid plate perforated with five axisymmetric air channels, in which Fabry-Perot resonances are used to boost the transmission through the lens. Lens B is identical to Lens A but concentric rings are added on both lens sides. The role of these rings is to generate additional resonances on the lens surface that, combined with the Fabry-Perot resonances, leads to a considerable amplification enhancement.

In both lenses, we consider two alternated kinds of channels: straight channels (SCs) and curled channels (CCs). The use of curled channels reduces significantly the radial dimension of the lenses, an important feature in practical applicaitons. In the axial plane, the SCs are defined by a width $a=5 \mathrm{~mm}$ and length $b=25 \mathrm{~mm}$, as indicated in the right panel of Fig. 1(a). The CCs have the same width $a$ than the SCs and can be characterized in the long wavelength regime $(\lambda \gg a)$ by an effective length $b_{\text {eff }}$, which is approximately equal to the 
(a)

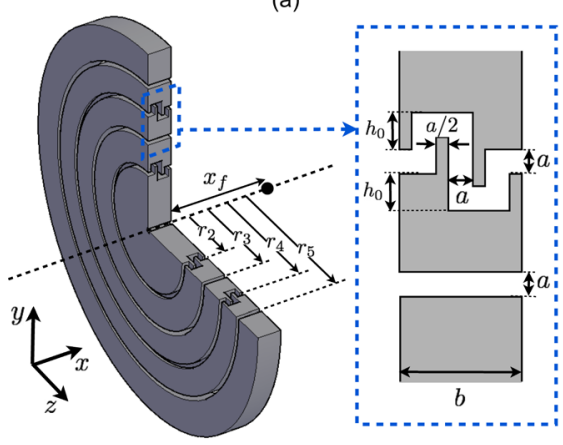

(c)

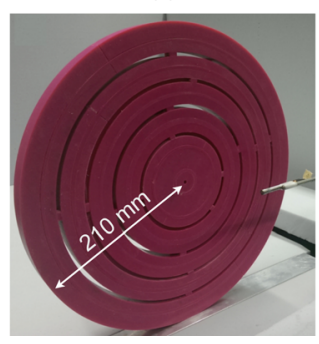

(b)

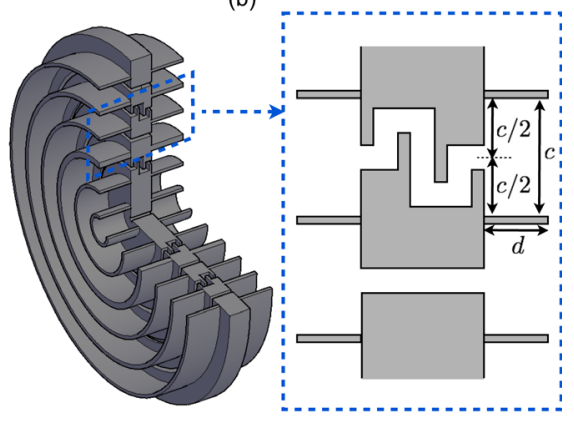

(d)

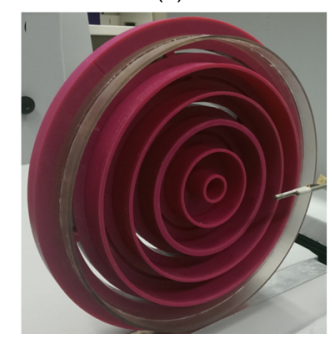

FIG. 1. (a) Lens A is composed of a circular plate with five axisymmetric air channels. $x_{f}$ is the focal length and $r_{2}$ to $r_{5}$ denote the central radius of the channels $\left(r_{1}=0\right.$ is the radius of the central channel). The panel on the right shows in detail the geometry of the air channels in the axial plane. (b) Lens B is identical to Lens A, but concentric rings are added on both sides of the lens. The panel on the right shows the geometry of the cavities formed by the rings in the axial plane. (c) and (d) are pictures of the 3D printed Lens A and Lens B, respectively. shortest path taken by the wave to pass through the channel. ${ }^{19,20}$ For simplicity, $b_{\text {eff }}$ is controlled by a single parameter, $h_{0}$, that represents the height of the corrugations, see Fig. 1(a).

Fabry-Parot resonances that boost the transmission appear at wavelengths such that $\lambda \approx 2 b / n$ in the case of the SCs, or $\lambda \approx 2 b_{e f f} / n$ in the case of the CCs, with $n$ being a positive integer. Thus, the global transmission is maximized if $b_{\text {eff }}=2 b$, in such a way that the first resonance in the SCs coincides with the second resonance in the CCs at wavelength $\lambda_{0} \approx 2 b=b_{\text {eff }}$. At resonance, the phase shift between incident and transmitted waves is expected to be approximately $\pi$ (half wavelength) in the SCs and zero (one wavelength) in the CCs, which means that waves emerging from two consecutive channels are out of phase. From these considerations, it follows that the lenses generate constructive interference at the focal point if the separation distance between two consecutive channels is such that

$$
d_{i}-d_{i-1}=\frac{\lambda_{0}}{2}
$$

where $d_{i}=\left(x_{f}^{2}+r_{i}^{2}\right)^{1 / 2}, i=1,2, \ldots, 5$ is the distance from channel $i$ to the focal point, with $x_{f}$ the focal length and $r_{i}$ the mean radii of the channels, see Fig. 1(a). From Eq. (1), we obtain the radii of channels $i=2, \ldots, 5$ as

$$
r_{i}=\sqrt{\left(\lambda_{0} / 2+\sqrt{x_{f}^{2}+r_{i-1}^{2}}\right)^{2}-x_{f}^{2}}
$$

where $r_{1}=0$ is the radius of the central channel. Note that the term $\lambda_{0} / 2$ in Eqs. (1) and (2) comes from the fact that waves emerging from two consecutive channels are out of phase. If only identical channels are considered, no phase shift exists between consecutive channels and the term $\lambda_{0} / 2$ should be replaced by $\lambda_{0}$, which would significantly increase $(\sim 40 \%)$ the lens radial dimension.

To determine the lens geometry, it is necessary to know the resonance wavelength $\lambda_{0}$ and the value of $h_{0}$ that makes SCs and CCs resonate at the same frequency. To obtain an initial estimation of these parameters, we studied separately the transmission properties of a periodic grating composed of SCs and another one composed of CCs. The transmission through the grating was calculated using modal expansions. ${ }^{21}$ The grating period in both cases is $b$. We note that these configurations do not reflect the actual lens geometry, in which CCs and SCs are alternated and their separation distance is not the same. However, since the Fabry-Parot resonances mainly depend on the channels dimensions and they are the same in all cases, we assumed that the obtained values of $\lambda_{0}$ and $h_{0}$ would reflect the actual behaviour of the channels in the lens. Solid lines in Figs. 2(a) and 2(b) show, respectively, the squared modulus and unwrapped phase of the transmission coefficient $T_{0}$ of the fundamental Bloch mode in the SC grating, in the frequency range studied $(0-10 \mathrm{kHz})$. A total transmission peak is observed at $f_{0}=$ $5805 \mathrm{~Hz}\left(\lambda_{0}=59.1 \mathrm{~mm}\right)$. Dashed lines in Figs. 2(a) and 2(b) show the results for the CC grating obtained by adjusting $h_{0}$ such that the second resonance peak matches the peak observed in the SC grating. The obtained value is $h_{0}=8.5 \mathrm{~mm}$. Note in Fig. 2(b) that at the resonance frequency, $f_{0}$, the phase difference of $T_{0}$ between SCs and CCs is $\pi$, as predicted before.

To obtain the optimal values of $\lambda_{0}$ and $h_{0}$ in the lens, we have calculated the lens amplification as a function of frequency and $h_{0}$, around the values obtained before $\left(f_{0}=5805\right.$ $\mathrm{Hz}$ and $\left.h_{0}=8.5 \mathrm{~mm}\right)$. The amplification is defined as $A=\left|p_{f} / p_{0}\right|^{2}$, where $p_{f}$ is the pressure at the focal point and $p_{0}$ is the pressure of the incident plane wave. The amplification in decibels is given by $A_{\mathrm{dB}}=10 \log _{10}(A)$. The channel radii were calculated using Eq. (2), with $\lambda_{0}=59.1 \mathrm{~mm}$ and the focal length arbitrarily fixed to one wavelength, $x_{f}=\lambda_{0}$. The resulting radii are $r_{2}=66.4 \mathrm{~mm}, r_{3}=102.3 \mathrm{~mm}$, $r_{4}=136.1 \mathrm{~mm}$, and $r_{5}=168 \mathrm{~mm}$. Finite elements simulations were performed using COMSOL Multiphysics in axisymmetric domain. Air was modelled as a viscothermal fluid to take into account the losses in the channels, and perfectly matched layers were used to simulate anechoic boundary 
(a)
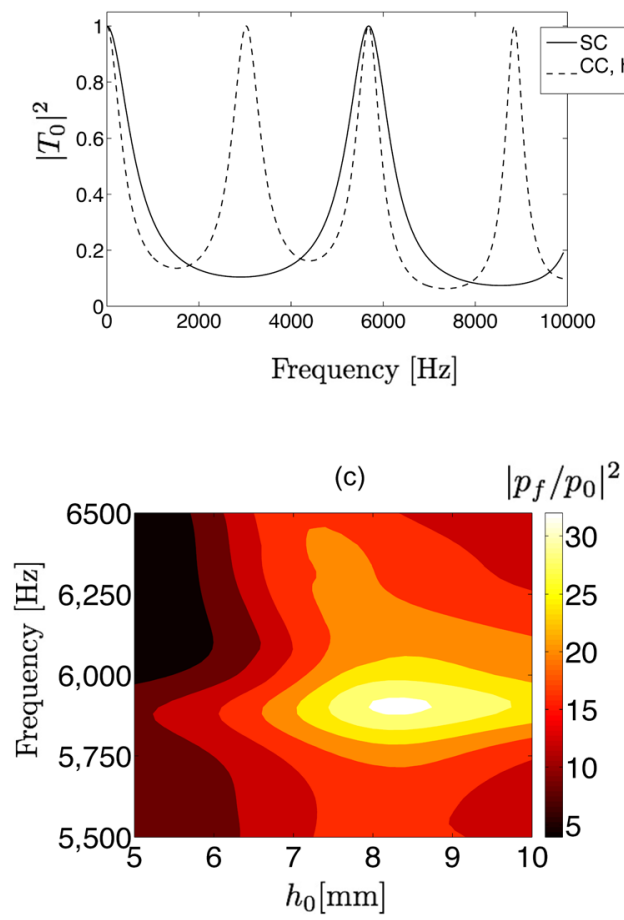

(b)

(b)

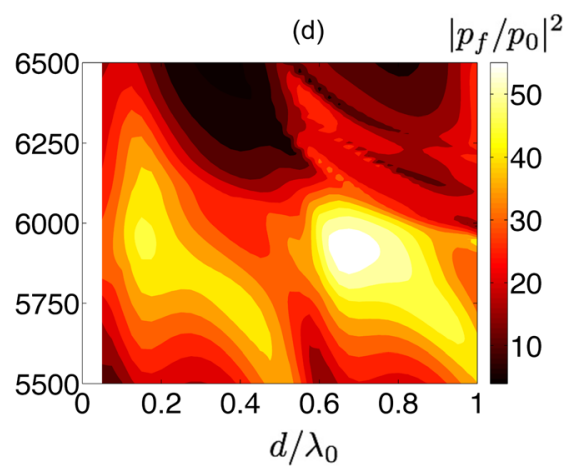

FIG. 2. (a) Modulus and (b) phase of the transmission coefficient of the first Bloch mode of a periodic grating of SCs (solid lines) and CCs (dashed lines) with grating period $b$. (c) Intensity amplification of Lens A as a function of frequency and $h_{0}$. (d) Amplification of Lens B as a function of frequency and $d / \lambda_{0}$. conditions. The $A$ vs. $f$ vs. $d$ plot is displayed in Fig. 2(c). The figure shows a maximum at precisely $h_{0}=8.3 \mathrm{~mm}$ and $f=5850 \mathrm{~Hz}$, very close to our preliminary estimation. At these values, the amplification is $A=30.7\left(A_{\mathrm{dB}}=14.9 \mathrm{~dB}\right)$.

Previous studies on extraordinary transmission showed that Fabry-Parot resonances can be combined with other resonant mechanism, as surface waves or cavity resonances, to further enhance the transmission through the structure. ${ }^{22}$ In the same direction, we added concentric rings to excite localized resonances in correspondence to each channel entrance [see the schematic diagram for Lens B in Fig. 1(b)]. In the axial plane, the rings form cavities centered around the channels. The width and height of the cavities is denoted by $c$ and $d$, respectively. In the case of narrow cavities, $d \gg c$, resonances appear at wavelengths such that $d / \lambda \approx(2 n-1) / 4$, with $n$ a positive integer. ${ }^{23}$ For wider cavities $(d \approx c)$, as the ones we are considering here, these resonances are strongly damped due to radiation losses. This means that the cavity resonances are able not only to radiate energy efficiently but also to couple easily with incident waves. One can thus expect that if cavities and Fabry-Parot resonances coincide at $\lambda_{0}$, the incident energy on one side can be efficiently funnelled and radiated on the other side.

To corroborate this, we have calculated the amplification as a function of frequency and $d / \lambda_{0}$. In order not to increase excessively the lens thickness, the amplification was calculated in the range $d / \lambda_{0} \in(0,1)$. The cavity width is fixed to $c=\lambda_{0} / 2$. This value is the asymptotic separation distance between two channels, $r_{i}-r_{i-1}$, for $i$ big, see Eq. (2), and represents the maximum value of $c$ under the constrain that all cavities are identical, which maximises the coupling with incident and radiative waves. We observe two amplification maxima, one at $d / \lambda_{0}=0.17$ and the other one at $d / \lambda_{0}=0.67$, both at frequency $f=5920 \mathrm{~Hz}$. These maxima correspond to cavity resonances type $d / \lambda_{0}=1 / 4$ and $d / \lambda_{0}=3 / 4$, respectively, as indicates the fact that they are separated by $d / \lambda_{0}=0.5$. However, we note that the values of $d / \lambda_{0}$ are smaller due to the aforementioned radiation losses. At the first maximum, $d / \lambda_{0}=0.17$, the amplification is $A=45\left(A_{\mathrm{dB}}=16.5 \mathrm{~dB}\right)$, and at the second maximum, $d / \lambda_{0}=0.67$, it reaches $A=57.4\left(A_{\mathrm{dB}}=17.6 \mathrm{~dB}\right)$. These values are substantially higher, $1.6 \mathrm{~dB}$ and $2.7 \mathrm{~dB}$, respectively, than the maximum amplification obtained with Lens A. The higher amplification observed at $d / \lambda_{0}=0.67$ is explained by the fact that the quality factor of the cavity resonances (and thus their excitation amplitude) increases with the height-to-width ratio $d / c$ (see Ref. 23).

Besides the high amplification, another interesting aspect of the proposed design is that the resulting lenses are relatively thin. For instance, the thickness of Lens $\mathrm{A}$ is $0.43 \lambda$, and that of Lens B at the first maximum in Fig. 2(d) is $0.76 \lambda$. This is about 4.5 and 2.5 times thinner than other PC lenses achieving similar amplifications. ${ }^{5}$

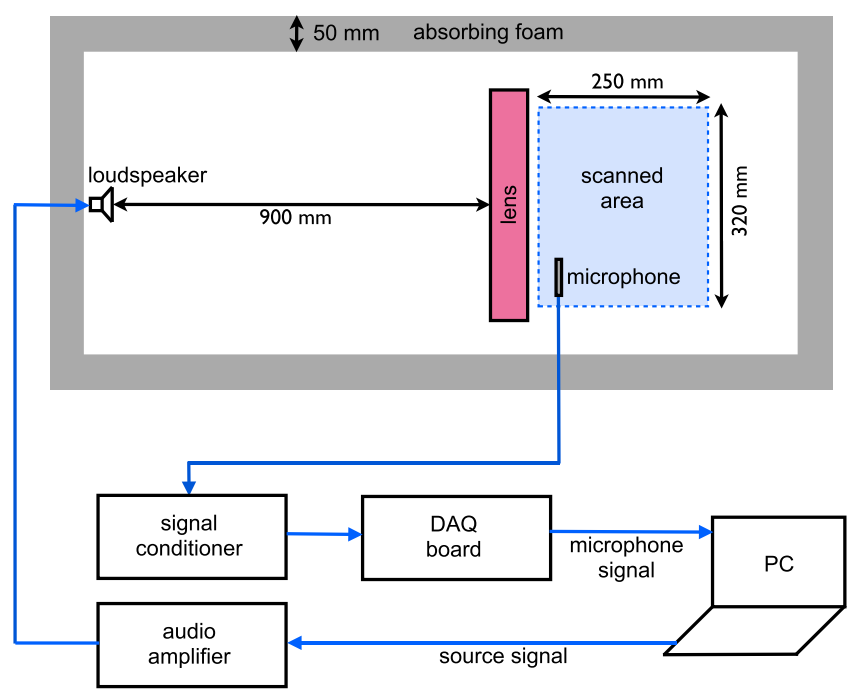

FIG. 3. Schematic diagram of the experimental setup and acquisition system. 
To validate our lens designs experimentally, we fabricated Lens A and Lens B by 3D printing (Stratasys Objet500). Figs. $1(c)$ and 1(d) show pictures of the experimental lenses. The material used was a rigid plastic (VeroMagenta RGD851) with manufacturer specified mass density $1.17-1.18 \mathrm{~g} / \mathrm{cm}^{3}$ and modulus of elasticity $2-3 \mathrm{GPa}$. The acoustic impedance in the material is 3 orders of magnitude bigger than in air, so that it can be considered as acoustically hard. Fig. 3 shows a schematic of the experimental setup. The lens was excited with a small $22 \mathrm{~mm}$ diameter loudspeaker (Clarion SRE 212H) placed on the lens axis at $900 \mathrm{~mm}$ from the left edge. The pressure on the right side was measured using a $1 / 4$ inch $(6.35 \mathrm{~mm})$ microphone set (G.R.A.S. 40BD) which position is controlled with a 2D stepper motor. The pressure field was scanned in the axial plane in a rectangular area of dimensions $250 \times 320 \mathrm{~mm}^{2}$ separated $20 \mathrm{~mm}$ from the right lens edge. The microphone signal was amplified using a PCB signal conditioner and digitalized using a National Instruments acquisition board. The pressure field was obtained using phase-sensitive detection to minimise the effect of noise on the experiments. The setup was enclosed in an absorbing box consisting of a $50 \mathrm{~mm}$ thickness melamine foam to minimise external reflections.

The left panels in Figs. 4(a) and 4(b) show the simulated scattered intensity fields, $\left|p / p_{0}\right|^{2}$ for Lens A and Lens B. The chosen frequency and geometrical parameters correspond to the amplification maxima in Figs. 2(c) and 2(d), respectivelly. The figures show a sharp focal spot with very high amplitude. The weak amplitude of the field on the left side puts in evidence the high efficiency of the lenses. The transmittance was computed numerically as the ratio of the
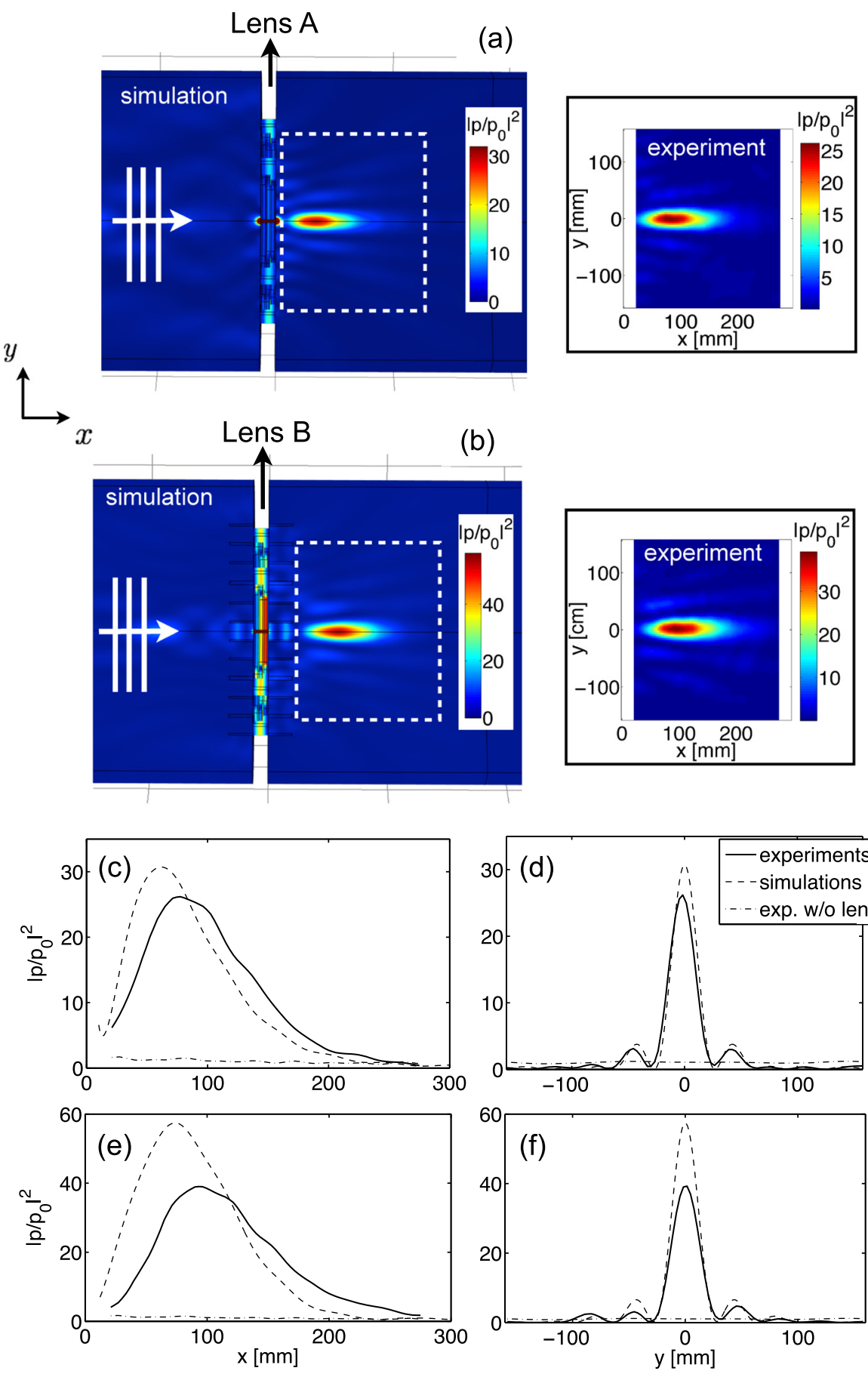
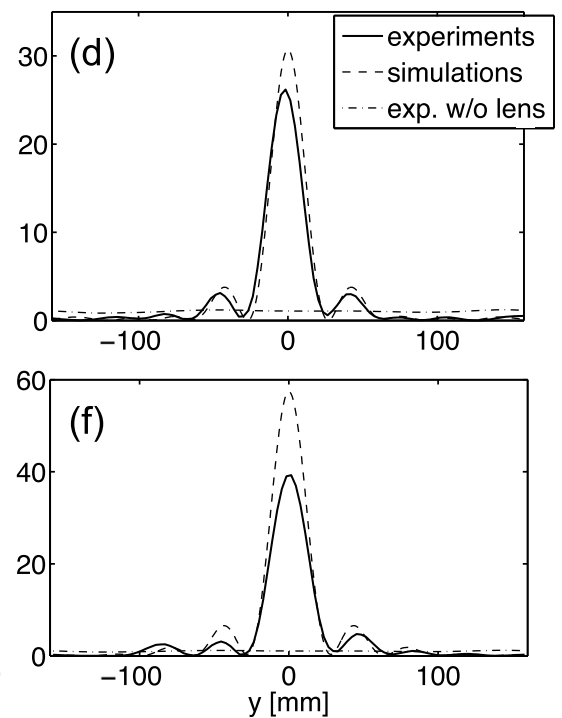

FIG. 4. Simulated (left panels) and experimental (right panels) intensity fields obtained with (a) Lens A and (b) Lens B. The dashed rectangle on the left figures represents the area in which the experimental field was measured. (c) and (d) represent the intensity fields along the $x-$ and $y-$ axis at focus obtained with Lens A. (e) and (f) represent the same quantities than (c) and (d) but obtained with lens B. $x=0$ indicates the right edge of the lens. 
transmitted to the incident intensity, obtaining $49 \%$ for Lens A and $83 \%$ for Lens B. Let us highlight that this efficiency is remarkably high, especially considering that the area occupied by the holes is only $14.2 \%$ of the total lens area.

For a more quantitative analysis, dashed lines in Figs. 4(c)-4(f) show the intensity fields along the $x$ - and $y$ - axes at the focal spot $\left(x=x_{f}\right)$. From these figures, we extract the focal length, measured from the right lens edge, and the full width at half maximum, FWHM. Since we are considering quadratic quantities, the FWHM is obtained from the width at quarter maximum of the intensity profiles in Figs. 4(d) and 4(f). The focal length of lens A is $x_{f}=61 \mathrm{~mm}$, in good agreement with the expected value $x_{f}=59.1 \mathrm{~mm}$. The focal length of lens B is $x_{f}=71 \mathrm{~mm}$. The fact that the focal length of Lens B is bigger than the expected value comes from the length correction typically applied to the actual length of resonant cavities (see, e.g., Ref. 24). Such correction can be regarded as a shift of the lens edge, which in turn generates a shift of the focal point. For what concerns the FWHM, we obtain $0.57 \lambda$ for lens $A$ and $0.61 \lambda$ for lens B.

The experimental intensity fields measured with Lens A and Lens B are displayed in the right panels of Figs. 4(a) and 4(b), respectively. Results are in good qualitative agreement with the numerical results; we observe a sharp focal spot, and the overall intensity map is very similar to the numerical one. As expected from the numerical analysis, we notice a substantial increase in the amplification of Lens $B$ with respect to Lens A. The amplification of Lens A is $A=26.2\left(A_{\mathrm{dB}}=14.2\right.$ $\mathrm{dB})$ while with Lens $B$ it reaches $A=39.5\left(A_{\mathrm{dB}}=16 \mathrm{~dB}\right)$. The amplification enhancement is therefore $2.8 \mathrm{~dB}$, which is consistent with the numerical results $(2.7 \mathrm{~dB})$.

Cutlines of the measured field along the $x$ - and $y$-axes are represented by solid lines in Figs. 4(c)-4(f). For comparison purposes, the field measured without the lens is also plotted in these figures (dashed-dotted lines). The experimental reference pressure $p_{0}$ is the mean value of the pressure field on the focal plane measured without the lens, i.e., the main value of the dashed-dotted line in Fig. 4(f). The experimental focal distances can be obtained using the lens maker equation $\frac{1}{x_{f}}=\frac{1}{S_{1}}+\frac{1}{S_{2}}$, where $S_{1}=900 \mathrm{~mm}$ is the distance from the source to the left lens edge and $S_{2}$ is the distance from the right lens edge to the image (focal spot). $S_{2}$ is obtained from the maxima in Fig. 4(e). The experimental focal distances are $x_{f}=71 \mathrm{~mm}$ for Lens A and $x_{f}=86 \mathrm{~mm}$ for Lens B, slightly higher than the numerical results, respectively $x_{f}=60 \mathrm{~mm}$ and $x_{f}=71 \mathrm{~mm}$. The measured FWHM is $0.61 \lambda$ for lens $\mathrm{A}$, and $0.65 \lambda$ for lens B, in good agreement with the simulations. This resolution is not as good as it can be obtained with metamaterial superlenses, ${ }^{25}$ but is close to the maximum achievable by conventional lenses, imposed by the diffraction limit $0.5 \lambda$.

The measured amplification is significantly lower than those observed numerically. Several reasons can have an influence in this discrepancy-for example, source-lens misalignments or dissipation. However, the main reason is the geometrical tolerance in the lens assembling. In the computation of the transmission spectra, we have observed that the resonance frequency of the channels is quite sensitive to $a$ and $h_{0}$, so that small deviations from the correct values can generate a detuning of the resonance frequencies of the channels. To corroborate this, we have measured the amplification in the focal point as a function of frequency (not shown here). We have observed that instead of a sharp peak at $f_{0}$, as obtained in the simulations [see Figs. 2(a) and 2(c)], the curve presented a wider high amplitude band between $5600 \mathrm{~Hz}$ and $6100 \mathrm{~Hz}$, indicating that the different channels resonate at slightly different frequencies around $f_{0}$. This uneven excitation of Fabry-Perot resonances can also explain the differences observed between the experimental and numerical focal distances.

In summary, we have proposed a lens design based on extraordinary acoustic transmission in binary Fresnel lenses. Experimental and numerical results are in good agreement and show the generation of a sharp focal spot and very high amplification (up to $16 \mathrm{~dB}$ experimentally). Furthermore, the resulting lenses are thinner than other designs reported previously in the literature. The focusing concept presented and tested in this paper can have potential application in medical diagnosis, acoustic imaging, or the design of directive acoustic sources.

The authors are grateful to Mythili Thevamaran for her help in the fabrication of the lenses. We acknowledge support from the US National Science Foundation (NSF) under Grant No. CMMI-1200319.

${ }^{1}$ A. Climente, D. Torrent, and J. Sánchez-Dehesa, Appl. Phys. Lett. 97, 104103 (2010).

${ }^{2}$ X. Yang, J. H. Page, Z. Liu, M. L. Cowan, C. T. Chan, and P. Sheng, Phys. Rev. Lett. 93, 024301 (2004).

${ }^{3}$ A. Håkansson, J. Sánchez-Dehesa, and L. Sanchis, Phys. Rev. B 70, 214302 (2004).

${ }^{4}$ A. Håkansson, F. Cervera, and J. Sánchez-Dehesa, Appl. Phys. Lett. 86, 054102 (2005).

${ }^{5}$ L. Sanchis, A. Yánez, P. L. Galindo, and J. Pizarro, Appl. Phys. Lett. 97, 054103 (2010).

${ }^{6}$ V. Romero-García, A. Cebrecos, R. Picó, V. J. Sánchez-Morcillo, L. M. Garcia-Raffi, and J. V. Sánchez-Pérez, Appl. Phys. Lett. 103, 264106 (2013).

${ }^{7}$ L. Zigoneanu, B. Popa, and S. A. Cummer, Phys. Rev. B 84, 024305 (2011).

${ }^{8}$ Y. Li, B. Liang, X. Tao, X. Zhu, X. Zou, and J. Cheng, Appl. Phys. Lett. 101, 233508 (2012).

${ }^{9}$ Z. Liu, X. Zhang, Y. Mao, Y. Y. Zhu, Z. Yang, C. T. Chan, and P. Sheng, Science 289, 1734 (2000).

${ }^{10} \mathrm{~J}$. Li and C. T. Chan, Phys. Rev. E 70, 055602 (2004).

${ }^{11}$ T. W. Ebbesen, H. J. Lezec, H. F. Ghaemi, T. Thio, and P. A. Wolff, Nature 391, 667 (1998).

${ }^{12}$ H. A. Bethe, Phys. Rev. 66, 163 (1944).

${ }^{13}$ F. J. Garcia-Vidal, L. Martin-Moreno, T. W. Ebbesen, and L. Kuipers, Rev. Mod. Phys 82, 729 (2010).

${ }^{14}$ M.-H. Lu, X.-K. Liu, L. Feng, J. Li, C.-P. Huang, Y.-F. Chen, Y.-Y. Zhu, S.-N. Zhu, and N.-B. Ming, Phys. Rev. Lett. 99, 174301 (2007).

${ }^{15}$ L. Zhou and G. A. Kriegsmann, J. Acoust. Soc. Am. 121, 3288 (2007).

${ }^{16}$ J. Christensen, L. Martin-Moreno, and F. J. Garcia-Vidal, Phys. Rev. Lett. 101, 014301 (2008).

${ }^{17}$ Y. Zhou, M.-H. Lu, L. Feng, X. Ni, Y.-F. Chen, Y.-Y. Zhu, S.-N. Zhu, and N.-B. Ming, Phys. Rev. Lett. 104, 164301 (2010).

${ }^{18}$ G. D'Aguanno, K. Q. Le, R. Trimm, A. Alù, N. Mattiucci, A. D. Mathias, and N. Aközbek, Sci. Rep. 2, 340 (2012).

${ }^{19}$ Z. Liang and J. Li, Phys. Rev. Lett. 108, 114301 (2012).

${ }^{20}$ Y. Li, B. Liang, X. ye Zou, and J. C. Cheng, Appl. Phys. Lett. 103, 063509 (2013).

${ }^{21}$ See supplementary material at http://dx.doi.org/10.1063/1.4896276 for details on the computation of the transmission coefficients.

${ }^{22}$ J. Christensen, L. Martin-Moreno, and F. J. Garcia-Vidal, Phys. Rev. B 81, 174104 (2010).

${ }^{23}$ C. Tam, J. Sound Vib. 49, 353 (1976).

${ }^{24}$ J.-P. Dalmont, C. Nederveen, and N. Joly, J. Sound Vib. 244, 505 (2001).

${ }^{25}$ X. Zhang and Z. Liu, Nat. Mater. 7, 435 (2008). 